\title{
The High-Velocity Clouds: Galactic or Extragalactic?
}

\author{
Hugo van Woerden ${ }^{1}$, Bart P. Wakker ${ }^{2}$, Ulrich J. Schwarz ${ }^{1}$, \\ Reynier F. Peletier ${ }^{1}$, and Peter M.W. Kalberla ${ }^{3}$ \\ 1 Kapteyn Institute, Postbus 800, 9700 AV Groningen, The Netherlands \\ 2 Department of Astronomy, University of Wisconsin, Madison WI, USA \\ 3 Radioastronomisches Institut der Universität, Bonn, Germany
}

\begin{abstract}
We present firm evidence that one of the major high velocity clouds (HVCs), Complex A, lies in the Milky Way Halo, at a vertical distance $z=3-7$ kpc from the Galactic plane. For clouds MII/MIII, Danly et al. and Keenan et al. had already found $z<5 \mathrm{kpc}$. We further report that the metallicity in the largest $\mathrm{HVC}$, Complex $\mathrm{C}$, is at least 0.1 solar. CaII/HI ratios in $6 \mathrm{HVCs}$, ranging from 0.002 to 0.07 times solar, set lower limits to their metallicities.

Blitz et al. have recently suggested that most of the HVCs are relatively unprocessed, extragalactic remnants of the gas which formed the Local Group of galaxies. However, the results mentioned above indicate that several major HVC complexes are neither primordial nor extragalactic. For the smaller HVCs, some of which have much higher velocities, a location in the Local Group remains a possibility.
\end{abstract}

\section{Introduction}

High velocity clouds (HVCs) are HI clouds with velocities inconsistent with simple differential galactic rotation. Their origins remain unclear; major candidates include a Galactic Fountain and accretion of intergalactic gas (see review by Wakker \& Van Woerden 1997).

Blitz et al. (1997) claim the HVCs are 'remnants of Local Group formation', and 'most plausibly explained as members of the Local Group of galaxies'. While no details of their work have been published, the main arguments of Blitz et al. appear to be the following. 1) The velocity centroid of the HVC ensemble is the same as that of the Local Group. 2) If the HVCs are gravitationally bound and tidally stable, their distances must be similar to those of Local Group galaxies. 3) The angular sizes and LSR velocities of HVCs appear to be inversely correlated. These arguments are all of a statistical nature and hence, even if applicable in general, carry no weight for individual HVCs. Moreover, the conditions for the second argument may not be fulfilled. What is required is direct distance determinations for individual HVCs - a program we have pursued over the last several years.

The only direct method available is to search for interstellar absorption at the HVC's velocity in the spectra of stars at different distances. Schwarz et al. (1995) have discussed the method, and its pitfalls, in detail. In view 
of the uncertain abundance of metal ions in HVCs, and their strong small scale structure (Wakker \& Schwarz 1991), we require a combination of high resolution optical (or ultraviolet) spectra of both stellar and extragalactic probes with $\mathrm{HI}$ column density measurements at high angular resolution.

The CaII/HI abundance ratios measured for 6 HVCs (Wakker \& Van Woerden 1997) range between 0.002 and 0.07 times the total solar Calcium abundance. Given the unknown degree of ionization of Calcium, its depletion onto interstellar grains, and the possible destruction of grains in interstellar shocks, these CaII abundances give loose lower limits only to the total Calcium abundances in HVCs. Abundances of various other metal ions indicate metallicities of order 0.1 in several HVCs (Wakker \& Van Woerden 1997).

\section{Distance Limits for Three Major HVC Complexes}

For Complex M, Danly et al. (1993) and Keenan et al. (1995) found a distance $d<5 \mathrm{kpc}(z<4.4 \mathrm{kpc}$ above the plane) from absorptions in a stellar probe spectrum, and abundances $>0.15$ solar for SiII and 0.02 solar for CaII. (Note that this distance limit applies to clouds MII/MIII only. Cloud MI has a different velocity, is not connected, and may be at a different distance.)

For Complex H, Wakker et al. (1997) have analyzed IUE spectra of OB stars at distances $1.3-5.0 \mathrm{kpc}$. The lack of MgII, CII and OI absorptions shows that Complex $\mathrm{H}$ has $d>3.5 \mathrm{kpc}$, and probably $d>5 \mathrm{kpc}$. (Because of its low latitude, the limits on $z$ are insignificant.) Since no extragalactic probe spectrum was available, no abundances could be derived; however, if Complex $\mathrm{H}$ were not beyond the stellar probes. the non-detections of absorption would imply extremely low abundances (factors $10^{2}-10^{5}$ below solar).

For the largest HVC, Complex $C$, two extragalactic probes have given CaII abundances of 0.008 and 0.013 times solar (Wakker et al. 1996b). Recent HST spectra of Mark 290 by Wakker suggest an SII abundance of order 0.10 solar. Several UV and CaII studies of stars at distances $d$ up to $2.5 \mathrm{kpc}$ have yielded $z>1.7 \mathrm{kpc}$ (Wakker \& Van Woerden 1997). Recent observations with WIYN by Wakker and with the WHT by Van Woerden \& Peletier of stars at distances $d$ up to $5-8 \mathrm{kpc}$ even indicate $z>3-4 \mathrm{kpc}$, implying an HI mass exceeding $10^{6} \mathrm{M}_{\odot}$ for this huge complex.

\section{Complex A: the First Firm Distance Bracket}

Complex A, the first HVC discovered, has often served as prototype for HVCs. HST spectra of the Seyfert galaxy Mark 106 show strong MgII absorption (Wakker et al. 1996a), indicating a MgII abundance $>0.03$ solar. The lack of high velocity $\mathrm{MgII}$ absorption in a neighbouring star, at distance $d=4 \mathrm{kpc}$, implies $z>2.5 \mathrm{kpc}$ for the distance of this HVC above the plane, unless its MgII abundance would vary by more than a factor 20 over a few degrees. 


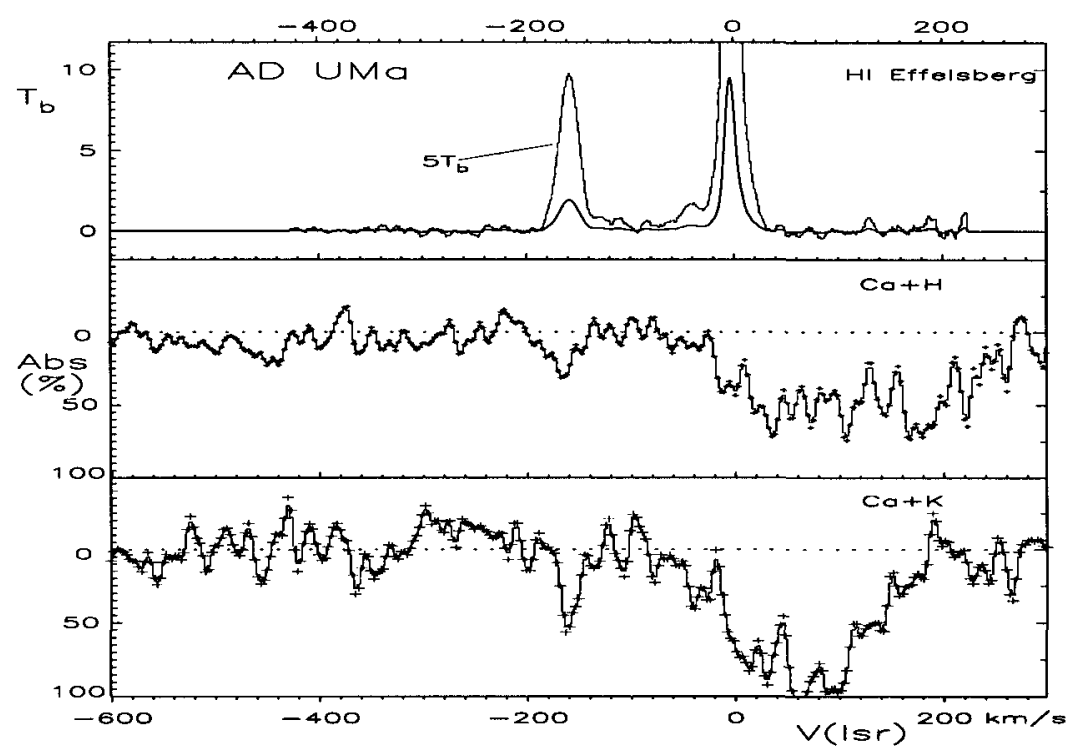

Fig. 1. HI emission and CaII absorption measured towards the RR Lyr variable AD UMa, which lies at $10 \mathrm{kpc}$ distance, projected on HVC Complex A (Van Woerden et al., in preparation). Velocities are given relative to the local standard of rest. The Effelsberg $21 \mathrm{~cm}$ profile, shown on two intensity scales, different by a factor 5 , has three components; that at $-160 \mathrm{~km} / \mathrm{s}$ is due to the HVC. The CaII $\mathrm{H}$ and $\mathrm{K}$ lines, measured with the William Herschel Telescope at La Palma $(10 \mathrm{~km} / \mathrm{s}$ resolution) both show, in addition to a broad stellar component, and $\mathrm{H}$-epsilon near the CaII $\mathrm{H}$-line, the same three interstellar components as the $21 \mathrm{~cm}$ line. This proves that HVC Complex A, as well as the other two clouds, lies in front of the star.

Recent spectra of the RR Lyr star AD UMa (distance $d=10 \mathrm{kpc}$ ), which lies very close to Mark 106, display strong absorption by the HVC in both CaII lines (Fig. 1). Together with the previous result, this sets a firm bracket of $2.5<z<7 \mathrm{kpc}$ on the distance of Complex A from the plane. The corresponding $\mathrm{HI}$ mass lies between 1.6 and $10 \times 10^{5} \mathrm{M}_{\odot}$.

\section{Conclusions and Prospects}

Two major HVC complexes, A and M, have now been firmly found to lie within our Galaxy. Both these and several other HVCs have significant metal abundances, showing that they do not consist of primordial gas.

What does the location of Complex A, high in the Galactic halo, tell us about its origin? Its pronounced small scale structure (Wakker \& Schwarz 1991) may be consistent with formation through instabilities in a Galactic Fountain. On the other hand, if Complex A originated in accretion of inter- 
galactic gas, possibly of Magellanic debris, the time scales of $10^{7}$ years derived for changes in its small scale structure (Wakker \& Van Woerden 1997) would require these to have developed close to their present location, probably by interaction with high- $z$ halo gas. The CaII and MgII abundances found allow a low-metallicity intergalactic origin; but in view of the uncertainties mentioned in Section 2, a Fountain origin may also be possible. In either case, a high halo temperature will be required to bring the gas to heights $z>3 \mathrm{kpc}$ (Houck \& Bregman 1990).

For two other large HVCs, Complex $\mathrm{C}$ and $\mathrm{H}$, only lower distance limits are available, leaving greater uncertainty concerning their location and origin. Further spectra of more distant stars may solve the problem, perhaps. Further work is also required for the many smaller HVCs, including the very high velocity clouds (VHVCs), which indeed may very well be intergalactic. But for these it will be much harder to find suitable probes.

We conclude that some HVCs lie within the Galaxy, that others may lie outside, and that further hard work on distance determinations is required.

Acknowledgements. The William Herschel Telescope is operated by the Royal Greenwich Observatory, in the Observatorio del Roque de los Muchachos of the Instituto de Astrofisica de Canarias. We thank the NFRA Programme Committee, and in particular Huib Henrichs, for their support of our program. The Effelsberg Telescope belongs to the Max Planck Institute for Radio Astronomy in Bonn. Wakker was partly supported by NASA through grant GO-04536.01-92A from STScI, which is operated by AURA, Inc., under NASA contract NAS5-26555. Wakker also thanks Blair Savage for financial support and useful discussions. HvW thanks Dieter Breitschwerdt, the IAU and the Leids Kerkhoven-Bosscha Fonds for support of his participation in this meeting.

\section{References}

Blitz L., Spergel D.N., Teuben P.J., Hartmann L., \& Burton W.B. (1997): BAAS $28,1349$.

Danly L., Albert C.E., \& Kuntz K.D. (1993): ApJ 416, L29.

Houck J.C., \& Bregman J.N. (1990): ApJ 352, 506.

Keenan F.P., Shaw C.R., Bates B., Dufton P.L., \& Kemp S.N. (1995): MNRAS 272,599 .

Schwarz U.J., Wakker B.P., \& van Woerden H. (1995): A\&A 302, 364

Wakker B.P., \& Schwarz U.J. (1991): A\&A 250, 484.

Wakker B.P., \& van Woerden H. (1997): ARA\&A 35, in press.

Wakker B.P., Howk C., Schwarz U.J., van Woerden H., Beers T.C., Wilhelm R., Kalberla P.M.W., \& Danly L. (1996a): ApJ 473, 834.

Wakker B.P., van Woerden H., Schwarz U.J., Peletier R.F., \& Douglas N.G. (1996b): A\&A 306, L25.

Wakker B.P., van Woerden H., de Boer K.S., \& Kalberla P.M.W. (1997): ApJ, submitted. 